\title{
Performance of halogen-free flame retardant EVA/MH/LDH composites with nano-LDHs and MH
}

\author{
DING YanYan ${ }^{1 *}$, XU Liang ${ }^{2} \&$ HU GengSheng ${ }^{1}$ \\ ${ }^{1}$ College of Materials and Environmental Engineering, Hangzhou Dianzi University, Hangzhou 310018, China; \\ ${ }^{2}$ Jinhua Fire Detachment of Zhejiang Province, Jinhua 310014, China
}

Received August 6, 2010; accepted February 28, 2011

\begin{abstract}
The flammability properties of three series of Ethylene-Vinyl Acetate/Magnesium Hydroxide/Layered Double Hydroxide (EVA/MH/LDH) composites as well as the flame retardance effect between LDH and the conventional flame retardant additive $\mathrm{MH}$ are investigated. In addition to acting as an insulating barrier, LDH assists the dispersion of $\mathrm{MH}$ particles in the EVA matrix, and promotes the formation of the char residues because of its abundant interlayer hydroxyl groups. Composites containing LDH show good flame retardancy in Cone and LOI testings. This means that the combustion properties of the composites have decreased, which may lessen the fire hazard when this materials are used.
\end{abstract}

layered double hydroxide, cone calorimeter, flame retardancy, EVA, synergistic effects

Citation: Ding Y Y, Xu L, Hu G S. Performance of halogen-free flame retardant EVA/MH/LDH composites with nano-LDHs and MH. Chinese Sci Bull, 2011, 56: 3878-3883, doi: 10.1007/s11434-011-4837-9

Ethylene-vinyl acetate copolymers (EVA) with different vinyl acetate (VA) contents are extensively used in many fields, especially in the cable industry as excellent insulating materials with good physical and chemical properties [1]. However, EVA resins are particularly flammable and emit a large amount of smoke when burnt. Magnesium hydroxide is a conventional non-toxic, smoke-suppressing halogen-free flame retardant additive with a high decomposition temperature that is used in flame retardant polymeric materials. However, its flame retardant efficiency is low, so very large amounts must be used, leading to a sharp drop in the mechanical properties of the flame retardant materials. To minimize this effect, the dispersion of $\mathrm{MH}$ and its compatibility with the polymer matrix must be improved. The use of very small (hyperfine) particles and surface treatment of $\mathrm{MH}$ are two important methods to achieve this. The usual surface modifiers for $\mathrm{MH}$ are organic reagents or polymers, which could be detrimental to combustion properties such as the limiting oxygen index (LOI). Hyperfine magnesium

*Corresponding author (email: yyding@mail.ustc.edu.cn) hydroxide has been very effective at improving the flame retardance and mechanical properties of polymeric materials, but its dispersion in polymer matrices is very difficult [2].

In recent years, the intercalated or exfoliated polymer/ LDH nanocomposites have attracted great interest in the field of materials science, because of their excellent thermal stability, flame retardance, and physico-chemical properties [3-6]. In the present work, we use MH and organic modified LDH layers as a flame retardant system and study their synergistic effects on the morphology and structure, flame retardant properties and mechanical properties of EVA/MH/ LDH nanocomposites. X-ray diffraction (XRD), transmission electron microscopy (TEM), scanning electron microscopy (SEM), LOI, dynamic mechanical thermal analysis (DMTA), and cone calorimetry (Cone) tests are performed. The aim is to study the effects of adding LDH and $\mathrm{MH}$ to EVA resin (in particular its flame retardant and mechanical properties), and also to explore three difference synergistic systems containing MgAl-LDH, ZnAl-LDH, or $\mathrm{MgFe}-\mathrm{LDH}$. The main purpose of this study is to develop a new type of low smoke, halogen-free flame retardant 
polymeric composite which can then be applied to creat halogen-free flame retardant EVA insulated wire and cable.

\section{Materials and methods}

\subsection{Materials}

EVA (containing $28 \mathrm{wt} \%$ vinyl acetate, 28-05) was purchased from Beijing Organic Chemical Plant. Magnesium hydroxide (MH, HV5M) was kindly supplied by Wuhu Keyan Chemical Material Technology Development Co., Ltd. LDH was chemically synthesized in our laboratory. All chemicals were obtained from China Medicine (Group) Shanghai Chemical Reagent Corporation including magnesium nitrate $\left[\mathrm{Mg}\left(\mathrm{NO}_{3}\right)_{2} \cdot 6 \mathrm{H}_{2} \mathrm{O}\right]$, aluminum nitrate $\left[\mathrm{Al}\left(\mathrm{NO}_{3}\right)_{3} \cdot 9 \mathrm{H}_{2} \mathrm{O}\right]$, iron nitrate $\left[\mathrm{Fe}\left(\mathrm{NO}_{3}\right)_{3} \cdot 9 \mathrm{H}_{2} \mathrm{O}\right]$, sodium hydroxide $[\mathrm{NaOH}]$ and sodium dodecyl sulfate (SDS). All were analytically pure and used without further purification.

\subsection{Preparation}

MgAl-LDH was synthesized by co-precipitation. A mixed solution containing $\mathrm{Mg}\left(\mathrm{NO}_{3}\right)_{2} \cdot 6 \mathrm{H}_{2} \mathrm{O}$ and $\mathrm{Al}\left(\mathrm{NO}_{3}\right)_{3} \cdot 9 \mathrm{H}_{2} \mathrm{O}$ with a $\mathrm{Mg}^{2+} / \mathrm{Al}^{3+}$ mol ratio of 3:1 was added dropwise under $\mathrm{N}_{2}$ to a vigorously stirred solution of SDS until the $\mathrm{pH}$ reached 10.0. The resulting suspension was aged at $80^{\circ} \mathrm{C}$ for $8 \mathrm{~h}$ under $\mathrm{N}_{2}$. The slurry was washed thoroughly with deionized water and dried under vacuum at $70^{\circ} \mathrm{C}$ for $24 \mathrm{~h}$, to give $\mathrm{MgAl}-\mathrm{LDH}$.

$\mathrm{ZnAl}-\mathrm{LDH}$ was also synthesized by co-precipitation. A mixed solution containing $\mathrm{Zn}\left(\mathrm{NO}_{3}\right)_{2} \cdot 6 \mathrm{H}_{2} \mathrm{O}$ and $\mathrm{Al}\left(\mathrm{NO}_{3}\right)_{3}$. $9 \mathrm{H}_{2} \mathrm{O}$ with a $\mathrm{Zn}^{2+} / \mathrm{Al}^{3+}$ mol ratio of $3: 1$ was added dropwise under $\mathrm{N}_{2}$ to a vigorously stirred solution of SDS until the $\mathrm{pH}$ reached 10.0. The suspension was aged and the resulting slurry washed and dried as above to give $\mathrm{ZnAl}-\mathrm{LDH}$.

$\mathrm{MgFe}-\mathrm{LDH}$ was synthesized by ion-exchange. A reddish-brown solution of $\mathrm{Mg}\left(\mathrm{NO}_{3}\right)_{2} \cdot 6 \mathrm{H}_{2} \mathrm{O}$ and $\mathrm{Fe}\left(\mathrm{NO}_{3}\right)_{3}$. $9 \mathrm{H}_{2} \mathrm{O}$ with $\mathrm{Mg}^{2+} / \mathrm{Fe}^{3+}$ mol ratio of $3: 1$ was added dropwise under $\mathrm{N}_{2}$ to a vigorously stirred solution of $2 \mathrm{~mol} / \mathrm{L} \mathrm{NaOH}$ until the $\mathrm{pH}$ reached 9.5. The resulting reddish-brown suspension was aged at $100^{\circ} \mathrm{C}$ for $6 \mathrm{~h}$ under $\mathrm{N}_{2}$. The pristine LDH was then obtained by thoroughly washing and drying the slurry. Then a desired amount of SDS aqueous solution was added into an aqueous solution which containing $2.5 \mathrm{~g}$ pristine $\mathrm{LDH}$. This solution was stirred mechanically at $100^{\circ} \mathrm{C}$ for $7 \mathrm{~h}$ under $\mathrm{N}_{2}$, and the solid obtained was washed thoroughly and dried under vacuum for $48 \mathrm{~h}$, to give the final MgFe- LDH, which was a pale yellow solid.

All compositions were melt compounded using a two-roll mill at about $120^{\circ} \mathrm{C}$ for $15 \mathrm{~min}$. After mixing, the mixtures were then compression molded at about $120^{\circ} \mathrm{C}$ into sheets ( 1 and $3 \mathrm{~mm}$ thickness) under a pressure of $10 \mathrm{MPa}$ for 10 min. The 3-mm sheets were cut into specimens of suitable size for fire testing, while dumbbell-shaped samples for mechanical property tests were cut from the 1-mm sheets. The formulations used in the present work are listed in Table 1.

\subsection{Analyses and characterization}

$\mathrm{X}$-ray powder diffraction (XRD) analysis was performed on a Rigaku Japan K/max- $\gamma$ A X-ray diffractometer with $\mathrm{Cu} \mathrm{K \alpha}$ radiation (Toyko, Japan) $(\lambda=1.54178 \AA)$ at a scan rate of $0.02 \%$ s.

The transmission electron microscopy (TEM) images were obtained on a Hitachi H-800 transmission electron microscope (Tokyo, Japan) with an accelerating voltage of $200 \mathrm{kV}$. The sample was ultramicrotomed with a diamond knife on an LKB Pyramitome (Stockholm, Sweden) to give 80-100 nm thick slices.

An scanning electron micrograph (SEM) was used. The specimens were cryogenically fractured in liquid nitrogen, and then sputter-coated with a conductive layer.

The limiting oxygen index (LOI) measurements were carried out using an HC-2 instrument (China) according to ASTM D 2863.

A cone calorimeter (Stanton Redcroft Type, made in England) was used to measure the flammability characteristics of EVA and its blends under a heat flux of $35 \mathrm{~kW} / \mathrm{m}^{2}$ according to ISO5660. The parameters used for flammability characterization include the heat release rate (HRR), the time to ignition, etc.

Dynamic mechanical thermal analyses (DMTA) were performed on a Perkin Elmer Diamond DMA (Massachusetts, USA) at a constant frequency of $5 \mathrm{~Hz}$ and a heating rate of $5 \% \mathrm{~min}$.

\section{Results and discussion}

\subsection{Morphological characterization}

The XRD patterns in the range of $2 \theta=2^{\circ}-10^{\circ}$ for four composite samples are shown in Figure 1. The (003) and (006) characteristic diffraction peaks characteristic of the

Table 1 Details of the samples

\begin{tabular}{ccccc}
\hline Sample & EVA & EM0 & EM1 & EM2 \\
\hline Composition & EVA & EVA/MH & EVA/MH/MgAl & EVA/MH/ZnAl \\
Content $(\%)$ & 100 & $50 / 50$ & $50 / 45 / 5$ & $50 / 45 / 5$ \\
\hline
\end{tabular}




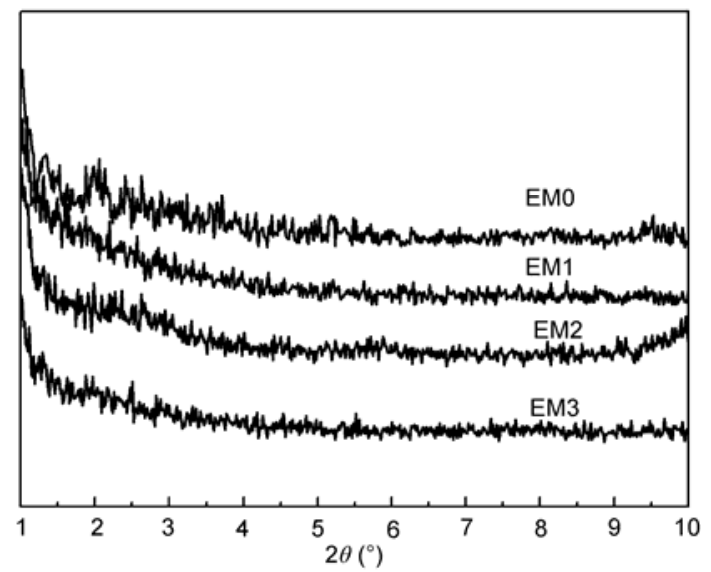

Figure 1 Small angle X-ray diffraction of composite samples.

nano-LDHs have completely disappeared, which indicates that the long range order in the inorganic phases has been completely destroyed and that the MgAl-LDH, ZnAl-LDH and $\mathrm{MgFe}-\mathrm{LDH}$ layers have been completely exfoliated inthe EVA matrix [7,8].

SEM images of EM0, EM1, EM2, and EM3 samples are shown in Figure 2. The MH particles in the sample EM0 are obviously badly agglomerated. Although the MH particles and LDH layers are difficult to discern in the SEM images, relatively well-dispersed $\mathrm{MH}$ particles as opposed to agglomerates are seen in the EM1, EM2, and EM3 samples, with sample EM1 being the most evenly distributed. The TEM images in Figure 3 also show that the distribution of inorganics in sample EM1 (Figure 3(b)) is more homogeneous than the distribution in sample EM0 (Figure 3(a)), which means that the addition of LDH improve to the distribution of $\mathrm{MH}$ into EVA. This may be because of the SDS within the LDH, which has hydrophobic functional groups that might help $\mathrm{MH}$ to disperse evenly in the EVA matrix. The LDHs could then act as dispersants during the melt blending of $\mathrm{MH}$ with EVA and thus help $\mathrm{MH}$ particles to disperse homogenously in the EVA [9].

It is well known that dispersion of inorganics in the polymer matrix will influence the properties of nanocomposites. Sample EM1 with more uniform dispersion should also perform best. It will be examined in the following tests.

\subsection{Dynamic mechanical thermal analysis}

DMTA gives accurate $T_{\mathrm{g}}$ (glass transition temperature) values of polymer. The effects of $\mathrm{LDH}$ on the $T_{\mathrm{g}}$ of the polymer composites are shown in Figure 4. The $T_{\mathrm{g}}$ values of three composite samples are lower than the $T_{\mathrm{g}}$ of EM0. It seems that the addition of LDH actually enhances the mobility of the polymer chain segments compared to the control EM0 sample. This is because the LDHs which contain SDS reduce the agglomerations of the $\mathrm{MH}$ particles, dispersing them more evenly in the EVA matrix [10]. The homogenously dispersed $\mathrm{MH}$ particles make the composites more flexibile with a lower $T_{\mathrm{g}}$. The curves of storage modulus of samples vs the temperature are shown in Figure 5. The storage modulus of sample EM0 is much larger than that of the pure EVA sample due to the high stiffness of the MH filler. It has been reported that the storage modulus of poly(propylene) also increased with increasing $\mathrm{MH}$ content [11]. However, the storage modulus of the EVA/MH/LDH samples is lower than that of EM0. This is also a result of better dispersion of $\mathrm{MH}$ in the presence of the $\mathrm{LDH}$, making EVA/MH/LDH samples more flexible than that of the EM0 sample without LDH.
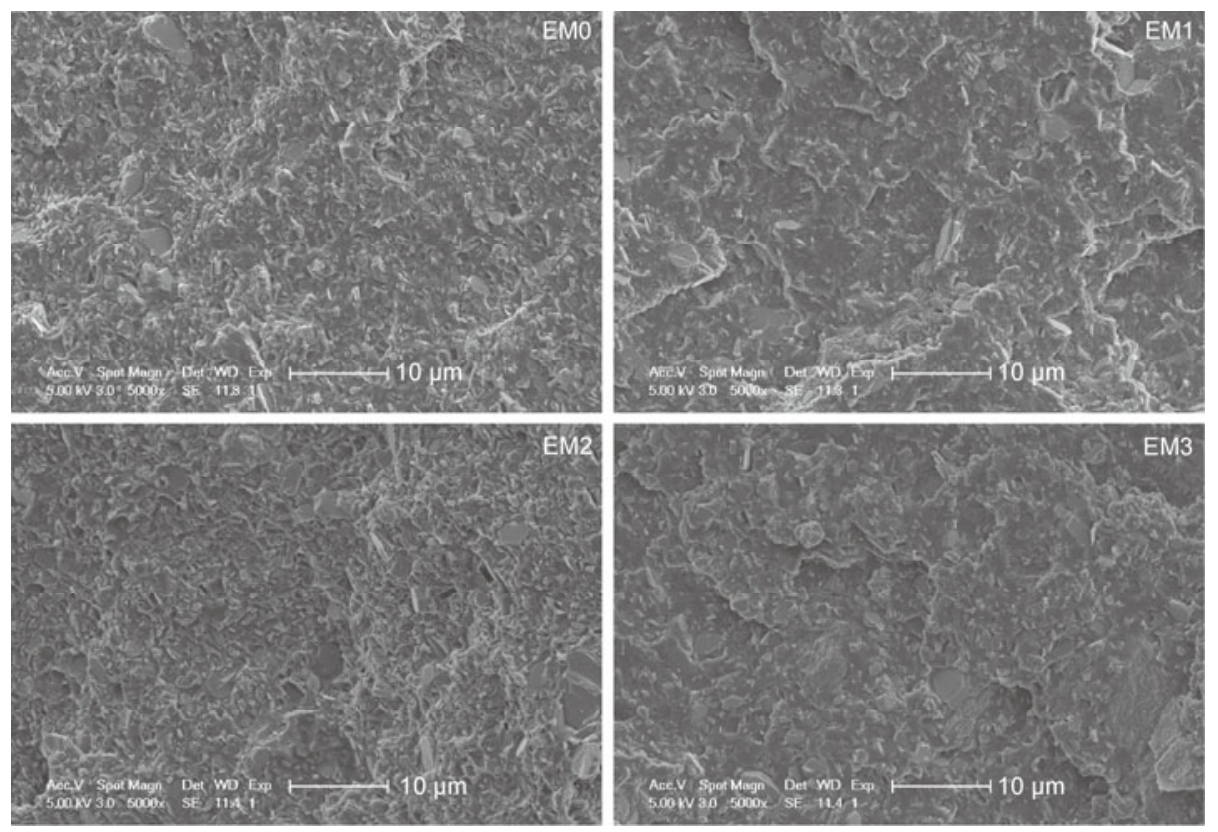

Figure 2 SEM photographs of EM0 and composite samples (scale $=10 \mu \mathrm{m})$. 


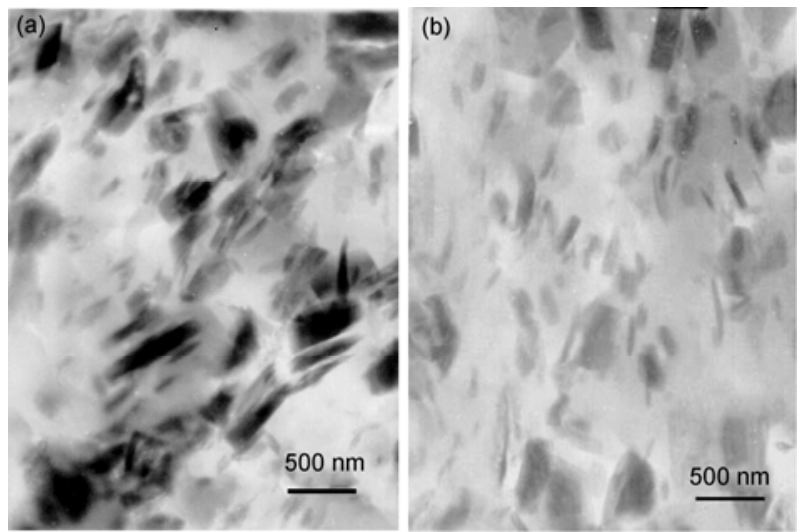

Figure 3 TEM photographs of EM0 (a) and EM1 (b) composites.

The LOI values of various samples with and without LDH are listed in Table 2. Substitution of LDH for $\mathrm{MH}$ increases the LOI values slightly compared with sample EM0 [12], with the greatest increase shown for sample EM1. These results are consistent with the TEM and SEM analyses and are further evidence that LDH can act as a synergistic flame retardant agent in the presence of $\mathrm{MH}$. Not only are the LDH layers effective barriers themselves, they also help the $\mathrm{MH}$ additive disperse more evenly in the EVA matrix and thus increase the LOI values compared to sample EM0.

Table 2 The LOI values of EVA, EM0 and nanocomposite samples

\begin{tabular}{cccccc}
\hline Sample & EVA & EM0 & EM1 & EM2 & EM3 \\
\hline LOI & 22 & 32 & 35 & 33 & 32 \\
\hline
\end{tabular}

A cone calorimeter is used to evaluate the fire properties of materials. The time to ignition $\left(t_{\text {ign }}\right)$, the heat release rate (HRR), the peak heat release rate (PHRR), and mass loss rate (MLR) were measured and are given in Table 3. The fire development test can be followed by plotting the HRR values as a function of time (Figure 6). The PHRR value of pure EVA is around $1860 \mathrm{~kW} / \mathrm{m}^{2}$. The PHRR of sample EM0 containing MH only is $553 \mathrm{~kW} / \mathrm{m}^{2}$, while the PHRRs of EM1, EM2 and EM3 samples decrease to 228, 403 and $281 \mathrm{~kW} / \mathrm{m}^{2}$, respectively. Sample EM1 displays a remarkable reduction in the PHRR of almost $60 \%$ relative to EM0. Composites containing of LDH show good flame retardancy based on Cone analysis with very different fire behavior relative to EM0 with only $\mathrm{MH}$. This means that the good dispersion of flame retardant in these samples and the synergistic effects of $\mathrm{LDH}$ and $\mathrm{MH}$ are efficient in inducing significant fire retardancy.

This differences can be easily understood from the appearance of the residues (Figure 7). While pure EVA is entirely burnt out, a thin layer of carbonaceous species is recovered after the combustion of EM0. Much more char is present after combustion of the LDH-containing composite samples, suggesting that the synergetic system helps char

Table 3 Cone calorimetry data for the samples

\begin{tabular}{lccccc}
\hline Cone calorimetry data & EVA & EM0 & EM1 & EM2 & EM3 \\
\hline$t_{\text {ign }}(\mathrm{s})$ & 55 & 113 & 128 & 105 & 122 \\
PHRR $\left(\mathrm{kW} / \mathrm{m}^{2}\right)$ & 1860 & 553 & 228 & 403 & 281 \\
$t_{\text {PHRR }}(\mathrm{s})^{\mathrm{a})}$ & 183 & 318 & 436 & 330 & 418 \\
MLR $(\mathrm{g} / \mathrm{s}$, average value $)$ & 0.153 & 0.098 & 0.07 & 0.095 & 0.07 \\
\hline
\end{tabular}

a) $t_{\text {PHRR }}$ : Time at PHRR.
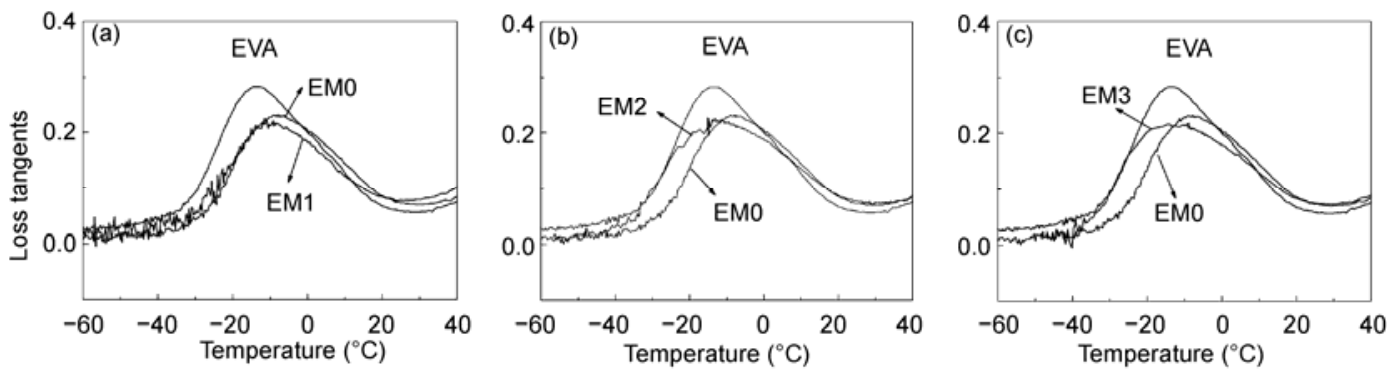

Figure 4 Loss tangents $(\tan \delta)$ of various samples vs. temperature.
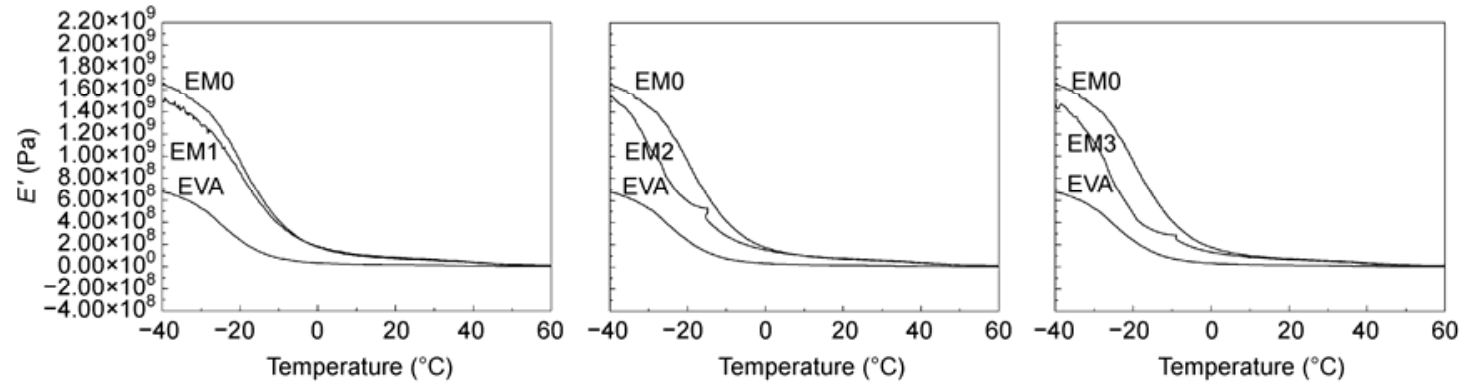

Figure 5 Storage modulus ( $E^{\prime}$ ) of various samples vs. temperature. 

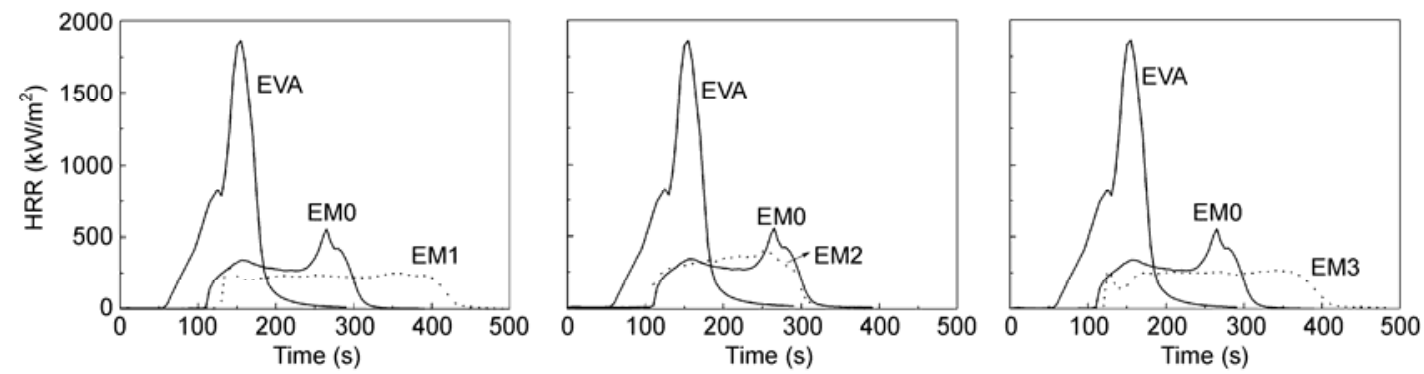

Figure 6 Heat release rate curves of EVA, EM0, and composites.
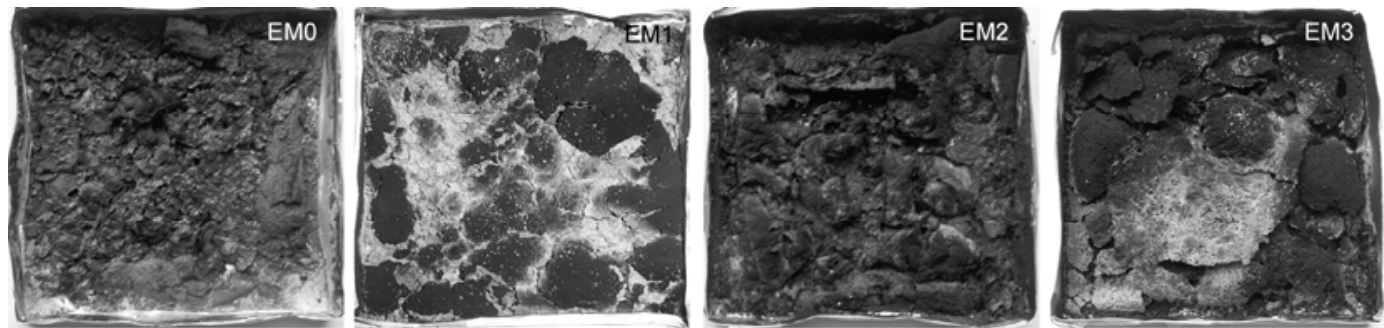

Figure 7 Digital photos of the char residues for EM0 and composite samples.

formation as the matrix degradation. The masses of char formed from the EM0, EM1, EM2, and EM3 samples are $1.1,2.7,1.6$, and $2.5 \mathrm{~g}$, respectively. Thus LDH has promoted the formation of the char residues due to the abundant hydroxyl groups in its interlayer space. Sample EM1 which contains very well dispersed LDH and MH (as shown by SEM and TEM analyses), formed a particularly homogeneous and cohesive char with some cracks and also gave the lowest HRR value.

As already discussed in the literature [13], the mechanism of the fire retardancy increase by the addition of nanolayers is believed to be a combination of barrier effects which retard the diffusion of the volatile products arising from polymer degradation and physical protection which keeps the material away from the flame. This mechanism will be most effective if the layers are homogeneously dispersed at the nanometer scale to form a cohesive char. In addition, the LDHs which contained SDS, help MH particles to disperse evenly in the EVA matrix and participate in the formation of char residue, further improving the flame retardancy.

\section{Conclusion}

The synergistic effects of organic-modified LDH with $\mathrm{MH}$ in flame retardant EVA/MH/LDH composites have been studied by XRD, TEM, SEM, LOI, DMTA, and Cone Calorimetry. The XRD data show that exfoliated LDH structures have been prepared in the EVA/MH/LDH composites. The SEM and TEM images reveal that the LDHs can help disperse the $\mathrm{MH}$ particles evenly in the EVA matrix. The DMTA shows that the $T_{\mathrm{g}}$ values of the composites decrease (relative to sample EM0 without LDH) with the introduction of $\mathrm{LDH}$ and approach the $T_{\mathrm{g}}$ of pure EVA. In other words, the introduction of LDHs makes the EVA/MH/LDH samples more flexible than sample EM0. The Cone calorimeter shows that EM1 displays a remarkable reduction in the PHRR of almost $60 \%$ relative to that of EM0. LDHs can promote not only the distribution of $\mathrm{MH}$ in composites, but also the formation of char residues to improve the flame retardant properties of composites in addition to the flame retardancy of LDH itself. The combustion properties of the composites are reduced, which may lessen the fire hazard of the materials in use.

This work was supported by the Zhejiang Province Education Department Research Projects (Y200908844) and the Schemes of Outstanding Young Teachers in Higher Education in Zhejiang Province.

1 Zhang Q L, Ma X Y, Wang Y F, et al. Morphology and interfacial action of nanocomposites formed from ethylene-vinyl acetate copolymers and organoclays. J Phys Chem B, 2009, 113: 11898-11905

2 Riva A, Canimo G, Fomperie L, et al. Fire retardant mechanism in intumescent ethylene vinyl acetate compositions. Polym Degrad Stab, 2003, 82: 341-346

3 Cavani F, Trifiro F, Vaccari A. Hydrotalcite-type anionic clays: preparation, properties and applications. Catal Today, 1991, 11: 173301

4 Zhang X, Lu J, Jin L, et al. Preparation of Rh-TPPTS complex intercalated layered double hydroxide and influences of host and guest compositions on its catalytic performances in hydroformylation reaction. Chinese Sci Bull, 2008, 5: 1329-1336

5 Ding Y Y, Xu L, Chu G H, et al. The thermal degradation studies of exfoliated poly(methyl methacrylate)/MgFe-LDH nanocomposites. Acta Polym Sin, 2009, 9: 942-948

6 Kotal M, Kuila T, Srivastava S K, et al. Synthesis and characterization of polyurethane/Mg-Al layered double hydroxide nanocomposites. J Appl Polym Sci, 2009, 114: 2691-2699

7 Chen W, Qu B J. Structural characteristics and thermal properties of 
PE-g-MA/MgAl-LDH exfoliation nanocomposites synthesized by solution intercalation. Chem Mater, 2003, 15: 3208-3213

8 Ding Y Y, Gui Z, Zhu J X, et al. Exfoliated poly(methyl methacrylate) $/ \mathrm{MgFe}$ layered double hydroxide nanocomposites with small inorganic loading and enhanced properties. Mater Res Bull, 2008, 43: 3212-3220

9 Kong Q H, Hu Y, Yang L, et al. Synthesis and properties of poly (methyl methacrylate)/clay nanocomposites using natural montmorillonite and synthetic Fe-montmorillonite by emulsion polymerization. Polym Composite, 2006, 27: 49-54

10 Laachachi A, Cochez M, Ferriol M, et al. Influence of $\mathrm{TiO}_{2}$ and $\mathrm{Fe}_{2} \mathrm{O}_{3}$ fillers on the thermal properties of poly(methyl methacrylate)
(PMMA). Mater Lett, 2005, 59: 36-39

11 Zhang G, Ding P, Zhang M, et al. Synergistic effects of layered double hydroxide with hyperfine magnesium hydroxide in halogen-free flame retardant EVA/HFMH/LDH nanocomposites. Polym Degrad Stab, 2007, 92: 1715-1720

12 Schafer A, Seibold S, Walter O, et al. Novel high T-g flame retardancy approach for epoxy resins. Polym Degrad Stab, 2008, 93: 557560

13 Lim S T, Hyun Y H, Choi H J. Synthetic biodegradable aliphatic polyester/montmorillonite nanocomposites. Chem Mater, 2002, 14: 1839-1844

Open Access This article is distributed under the terms of the Creative Commons Attribution License which permits any use, distribution, and reproduction in any medium, provided the original author(s) and source are credited. 